Cahiers $d u$ MONDE RUSSE

\section{Cahiers du monde russe}

Russie - Empire russe - Union soviétique et États indépendants

$50 / 2-3 \mid 2009$

L'Europe orientale, 1650-1730. Crises, conflits et renouveau

\title{
Meeting the Demands of ReasonJay BERGMAN
}

, Ithaca - Londres : Cornell University Press, 2009, XV-454 p.

\section{Wladimir Berelowitch}

\section{OpenEdition}

\section{Journals}

Édition électronique

URL : https://journals.openedition.org/monderusse/9796

DOI : $10.4000 /$ monderusse.9796

ISSN : $1777-5388$

Éditeur

Éditions de l'EHESS

Édition imprimée

Date de publication : 15 septembre 2009

ISBN : 978-2-7132-2260-3

ISSN : $1252-6576$

Référence électronique

Wladimir Berelowitch, " Meeting the Demands of ReasonJay BERGMAN », Cahiers du monde russe [En ligne], 50/2-3 | 2009, mis en ligne le 14 janvier 2013, consulté le 03 septembre 2022. URL : http:// journals.openedition.org/monderusse/9796 ; DOI : https://doi.org/10.4000/monderusse. 9796

Ce document a été généré automatiquement le 3 septembre 2022.

Tous droits réservés 


\section{Meeting the Demands of ReasonJay BERGMAN}

, Ithaca - Londres : Cornell University Press, 2009, XV-454 p.

Wladimir Berelowitch

\section{Jay BERGMAN, Meeting the Demands of Reason, The Life and Thought of Andrei Sakharov, Ithaca - Londres : Cornell University Press, 2009, XV-454 p.}

1 L'ouvrage de Jay Bergman constitue la biographie la plus récente de Saharov qui ait été publiée après la mort de ce dernier en décembre 1989. Au regard de celle qui fut écrite par Richard Lourie ${ }^{81}$, principalement fondée sur les Mémoires du dissident, elle est plus complète et mobilise des sources plus importantes : ce sont tous les écrits et interviews de Saharov, les mémoires et différentes mentions qui le font apparaitre, notamment dans les publications russes, ainsi que d'autres sources diverses. Jay Bergmann consacre aussi une part importante à l'itinéraire intellectuel de Saharov, s'attachant notamment à suivre sa pensée politique, depuis ses Réflexions sur le progrès, la coexistence et la liberté intellectuelle en 1968 (18 millions d'exemplaires vendus la première année de sa publication aux États-Unis, 56 éditions en 17 langues jusqu'en 1992) jusqu'à ses dernières interventions au Congrès des députés du peuple à Moscou, en décembre 1989.

2 Il est sans doute inutile de résumer les différentes parties de cet ouvrage, qui suit, comme il est normal, un plan strictement chronologique: la vie de Saharov est suffisamment connue et ses Mémoires suffisamment disponibles pour que cela ne soit pas nécessaire. Contentons-nous d'en marquer les grands tournants et les idées principales.

3 Avec raison, l'auteur insiste sur le lien entre Saharov - et la dissidence soviétique de façon générale - et l'intelligentsia du XIX siècle : le même refus intransigeant d'un ordre politique et juridique jugé inique et rétrograde, la même exigence morale qui peut aller jusqu'à une "manière de vivre». Mais il donne de l'intelligentsia un sens restreint classique (une catégorie qui se définit par son opposition au régime et qui, par conséquent, est révolutionnaire au moins en paroles), ce qui lui interdit, d'une part de 
tracer une continuité entre le $\mathrm{xIX}^{\mathrm{e}}$ siècle et la période soviétique, et d'autre part de rattacher Saharov à cette tradition, non pas seulement dans son évolution intellectuelle, mais dans ses origines mêmes. Ce choix est discutable: Saharov prend soin, dans ses Mémoires, d'évoquer son aïeul avocat qui avait signé un appel contre la peine de mort, nouant ainsi un fil généalogique important à ses yeux, et il nous paraît qu'il le fait avec raison. Quand bien même ce ne serait pas le cas, la fierté qu'il exprime en s'en réclamant devrait nous faire réfléchir. Plus généralement, l'opposition entre l'intelligentsia de l'époque impériale et celle de la période soviétique nous paraît largement artificielle: que ce soit sur le plan de l'histoire des catégories intellectuelles, de celle du statut des intellectuels vis-à-vis de l'État ou enfin de l'histoire des familles des intellectuels, les liens nombreux qui lient cette intelligentsia à l'ancienne nous obligent, nous semble-t-il, à dépasser les définitions "statistiques" de l'intelligentsia données par le régime soviétique.

4 L'auteur consacre plusieurs chapitres aux débuts de Saharov dans les études en physique, à son entrée à l'Institut de physique (FIAN) et au milieu des physiciens, enfin à son travail et à ses succès dans la fabrication de la bombe $\mathrm{H}$ dans l'installation, alors secrète, d'Arzamas-16 (à Sarov). Partie fouillée et documentée, dans laquelle, pour mieux comprendre l'exploit de Saharov, l'auteur a pris la peine d'éclairer les différents enjeux scientifico-militaires de la course aux armements. Il prend soin - certainement avec raison - de dégager Saharov de tout "syndrome Oppenheimer»: ce ne sont pas les remords (Saharov n'éprouvait nul doute sur l'utilité de son travail) mais d'autres ressorts qui ont poussé le physicien vers une attitude critique envers le régime.

5 L'entrée en dissidence de Saharov reste probablement le point le plus passionnant - et le plus mystérieux - de sa biographie : rappelons qu'il fut un des très rares membres de l'Establishment à rompre délibérément avec le régime, alors même que ce dernier fit tout pour le " garder ». Les traditions familiales mises à part (et que, comme on l'a vu, l'auteur écarte largement sinon légitimement), nous trouvons dans cette biographie trois ressorts. Le premier est l'exigence humanitaire et environnementale : des accidents survenus au cours des essais nucléaires de la bombe $\mathrm{H}$, l'inquiétude croissante de Saharov à propos des effets des retombées radioactives des essais en atmosphère le poussent à " entrer en politique». Le deuxième est d'ordre scientifique: c'est par une logique strictement professionnelle que Saharov empêche en 1964 l'élection à l'Académie d'un émule de Lysenko. Plus profondément - et c'est le sens du titre de l'ouvrage - ce sont des exigences de rationalité qui, tout à fait comme à l'époque des Lumières, poussent Saharov à inclure l'ordre juridique et politique dans une vision rationnelle d'un monde en progrès. Et pour finir, le ressort principal, toujours selon l'auteur, fut la dichotomie entre la position du physicien qui, en tant que scientifique et expert, est traité avec égards, en adulte, et celle du sujet soviétique, qui est traité comme un enfant irresponsable: déséquilibre que Saharov ressentit douloureusement à plusieurs occasions, notamment face à Hruščev. Sur ce fond, les aspects conjoncturels de la biographie de Saharov, et notamment sa rencontre et son second mariage avec Elena Bonner, certes importants, sont remis à leur place, finalement modeste. On pourrait ajouter que, sans doute - et l'auteur a raison d'insister là-dessus - le travail du physicien, qu'on sait hors du commun, lui ouvrit l'accès à une universalité de pensée à laquelle il prit goût et qu'il étendit à tous les aspects du monde, politique y compris.

6 L'analyse de l'action politique de Saharov, à partir de 1965-1968 qui sont les dates de naissance de la dissidence soviétique, et jusqu'à sa mort, est fouillée et convaincante. 
Parti de l'idée de convergence entre les « deux systèmes ", où une certaine naïveté et un sens réaliste du compromis avaient également leur part, Saharov aboutit à un rejet total du régime communiste, dont il réclame en 1989 l'effacement de la nouvelle constitution (puisque la constitution soviétique proclamait son rôle dirigeant dans son fameux article 6). «Radicalisation » qui épouse sans doute l'évolution de la dissidence, mais qui vient des idées de Saharov qui, selon l'auteur, émerge comme un véritable leader politique en 1989 et que sa propre évolution a conduit vers une "occidentalisation" totale et une détestation du régime soviétique. Du reste, l'auteur a raison de signaler que, malgré son engagement maximal dans les combats de la dissidence avec laquelle il partageait une haute exigence morale, Saharov a toujours gardé une position particulière qui lui venait à la fois de sa stature de grand scientifique, mais aussi, encore une fois, de sa vision universaliste, globale, du monde moderne.

Le dernier chapitre, consacré à l'action de Saharov depuis son retour de Gor'kij en décembre 1986, est un des plus intéressants, car il offre une synthèse de toute l'évolution politique du régime: les pages consacrées aux rapports entre Gorbačev et Saharov, notamment, sont très éclairantes. Dans la controverse qui, dans la littérature existante, entoure le rôle de la dissidence et la chute du régime soviétique, l'auteur adopte une position « optimiste » et crédite Saharov, notamment, d'avoir gravement influencé ses contemporains, en URSS notamment, et jusqu'à Gorbačev compris. Il cite des faits et des textes intéressants, bien que, malgré tout, ténus, à l'appui de cette hypothèse. Il est probable que nous soyons là en présence d'un débat impossible à trancher. Les idées de la dissidence ont agi plus par imprégnation que par emprunts directs (rappelons-nous l'épisode des décembristes) et à cet égard, il est certain que l'aura de Saharov fut très grande en Russie, comme l'atteste le véritable culte dont il a fait l'objet après sa mort : les enquêtes de Jurij Levada et de son équipe montraient qu'il était classé premier en 1990-1991 dans les sondages d'opinion parmi les personnalités qui avaient fait du bien au pays.

On pourrait reprocher à l'auteur sa méconnaissance des langues européennes autres que l'anglaise et la russe, ce qui appauvrit, malgré tout, sa bibliographie, de même que quelques coquilles (Landisbergis au lieu de Landsbergis...). Mais ce ne sont que des défauts mineurs : nous disposons, au total, d'une biographie complète et documentée de Saharov. Renouvelle-t-elle, fondamentalement, ce que nous savons de Saharov, notamment par ses Mémoires, du milieu des physiciens soviétiques, des projets atomiques, de la dissidence, de la perestroïka? Sans doute non, car l'ouvrage est trop centré sur la biographie de Saharov et les sources qui s'y attachent pour autoriser de semblables excursions, de sorte que ces questions passionnantes attendent encore leurs historiens futurs. Mais le moindre de ses mérites n'est pas de fournir une nouvelle matière à réflexion sur ces sujets. 


\section{NOTES}

81. Sakharov: a Biography, Hanovre : Brandeis University Press, 2002 (édition française : Richard Lourie, Sylvie Finkelstein, Sakharov : Une biographie, P. : Noir sur Blanc, 2005). 